\title{
CONTINUOUS INTRAVENOUS DRIP IN INFANTS AND CHILDREN
}

\author{
BY \\ WILLIAM C. BIACK, M.D. \\ (From the Department of Pediatrics, Stanford University School of \\ Medicine, San Francisco, California.)
}

Indications for continuous intravenous administration of fluids are perhaps more common in infants and children than in adults. The principal conditions in which the procedure is useful in paediatric practice are diarrhoea, persistent vomiting, severe burns, and states of severe dehydration and 'acidosis' associated with acute infections. The continuous intravenous drip used when the indications are definite is an invaluable therapeutic aid. It is the purpose of this paper to describe in detail the equipment and technique which give the most consistently good results. They vary only in a few details from the method described by Brush ${ }^{1}$.

\section{Description of equipment and technique}

\section{Materials and assembly :}

1250 c.c. graduated cylinder.

6 ft. black gum rubber transfusion tubing.

1 Murphy drip bulb.

1 connection tip to adapt the needle to tubing.

1 small calibre needle.

1 small, not graduated, plain ureteral catheter (No. 4 for babies, No. 5 or 6 for older children). 1 screw clamp.

A short piece (6-8 in.) of the tubing is connected to the bottom, of the graduated cylinder and the screw clamp slipped over the free end. Then the Murphy drip bulb and the rest of the tubing are attached. The needle connection tip is inserted into the lower end of the tubing and all the connections are tied with linen thread. New tubing requires special preparation before use to prevent reactions. This consists of two-hour boiling in 1 per cent. sodium citrate solution, rinsing in 1 per cent. ammonia and finally thorough washing with tap water. This apparatus is sterilized, except the catheter, by autoclaving or boiling. A needle should be chosen which fits snugly into the catheter. The needle should be cut off square about onehalf inch or a little less from the hub, and sterilized with heat. The catheter consists of a woven silk tube impregnated with a gummy distillate of linseed oil. It will not stand sterilization by boiling. Prolonged soaking is also deleterious. It is best sterilized by soaking it for fifteen minutes in a $1: 1,000$ solution of mercury oxycyanide. Following this it should with sterile precautions be flushed and washed with sterile water. This is most easily accomplished by means of a sterile Luer syringe and the cut needle 
described above. The catheter can be slipped over the needle by means of sterile forceps and flushed. Then it is placed in a dry, sterile, covered dish until dry. One catheter so prepared is sufficient for eight or more pieces of suitable length. Each piece as it is cut should be about two and a half inches long. If it is cut with sterile scissors the remainder of the catheter need not be sterilized again (fig. 1).

\section{Technique :}

A sterile tray containing the following should be prepared:-

1 scalpel.

2 mosquito clamps.

3 towel clips.

1 scissors.

$11 \frac{1}{2}$ c.c. Luer syringe.

1 hypodermic needle.

1 probe such as is used in dissection (for dilating the vein).

Yucca board, cotton, bandages, adhesive tape and novocaine should be available.

Success in the use of the continuous intravenous drip results from careful attention to certain details which in themselves are simple. In the following description an attempt has been made to include these details and to emphasize them properly. The rationale of their importance should be obvious if it is recognized at the onset that the aim of the procedure is to place the catheter in the vein with a minimum of trauma to the vein, a minimum of distortion to the natural course of the vein and to fasten the catheter in place in such a way that the child may move about and be moved without causing any change in the relation of the catheter to the veinwithout rotation, pulling, pushing, or side traction.

Because of its constant position in practically all individuals, and its situation on the lower extremity where the attachment of this apparatus causes a minimum of inconvenience both to the patient and to those attending him the great saphenous vein where it runs across the ankle just anterior to the medial malleous is the vein of choice.

Cut a piece of yucca board to conform roughly with the size of the foot and pad it well on one side with cotton held in place with roller bandage. Fasten this in turn to the outer side of the foot (the padding next to the skin) with adhesive tape and bandage which is carried up nearly to the medial malleolus. The purpose of this is two-fold; to limit the motion of the ankle and to afford a means of restraining the foot during the procedure. By means of adhesive tape, or preferably safety pins, fix the foot to the table or bed in a position of external rotation. Now fill the cylinder and tubing with warm solution for injection being careful to have the fluid in the Murphy drip bulb at the correct height.

Scrub the hands and put on sterile gloves. Prepare the skin with iodine and alcohol and drape with three sterile towels held in place with the towel clips. Anaesthetize the skin with novocaine. Make an incision $\frac{1}{2}$ inch long directly over and parallel with the course of the vein. The site of the incision may be previously marked by a pin scratch. The field should be brightly illuminated. Spread the tissue and strip the vein with the mosquito clamp. Then pass black silk ligatures under the upper and lower ends of the exposed vein but do not tie them. Lift the vein by means of the lower ligature and cut the vein about half in two with the scissors. If the vein is found to be quite small, dilation with the probe will facilitate entrance of the catheter. Further, if the end of the catheter itself is cut obliquely it will enter the vein more easily. In small subjects passage of the catheter 
occasionally requires a little patience but the vein is quite distensible and will accept a catheter which at first may appear to be much too large. The catheter should be wet before it is inserted and should be pushed into the vein about one inch. Now tie the proximal ligature firmly around the vein and catheter. It is well to anchor the ligature by bringing it back and tying it around the needle hub. The latter should be so placed in the

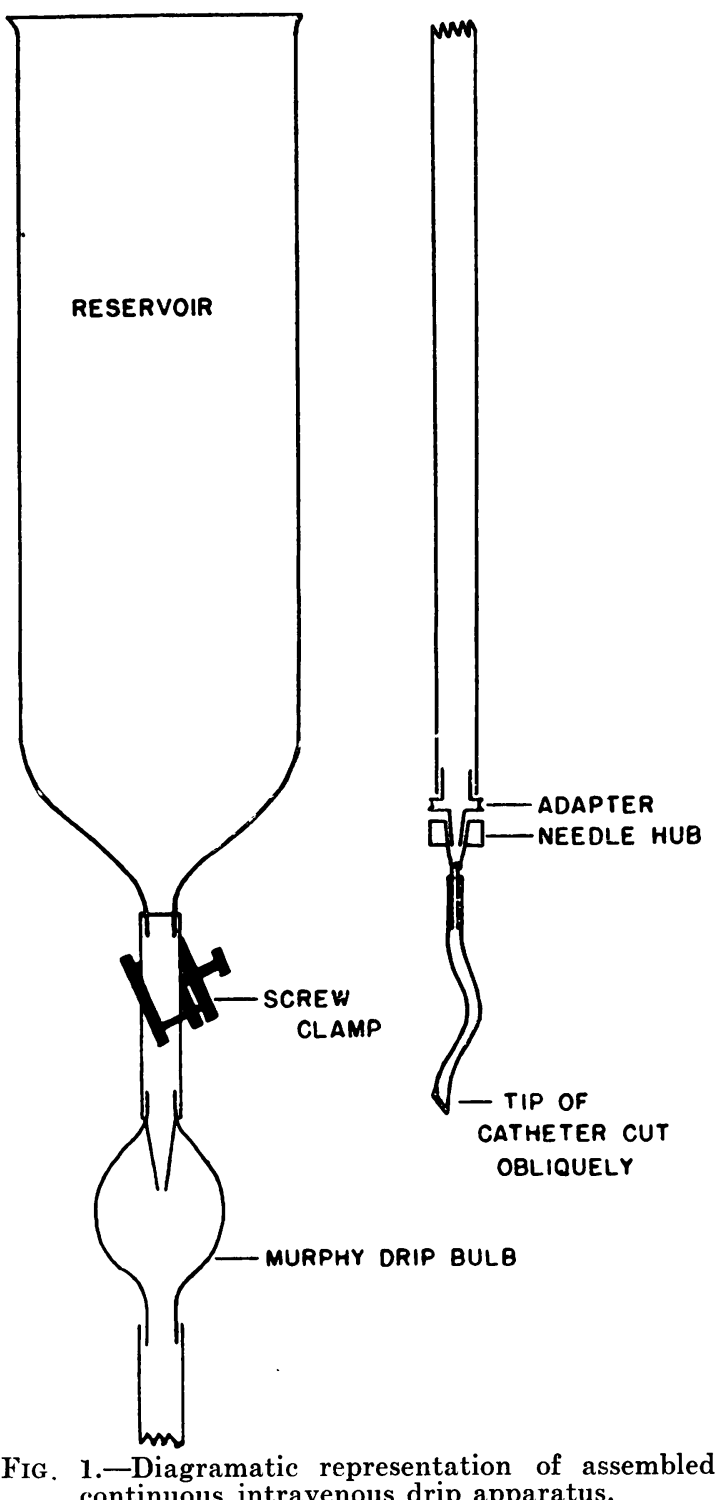

catheter that the flat side of the hub lies flat against the skin without any torsion of the catheter. Twisting the catheter twists the vein and will cause stoppage of flow. With a Luer syringe run in five or ten c.c. of sterile saline to prove the patency of the system. Then tie the lower ligature around the vein and cut one end short and one end long so that at the termination of the drip this ligature may be removed easily without opening the wound. 
Connect the previously filled tube with the needle and permit the fluid to drip in fairly rapidly. Fasten the needle hub firmly against the skin with adhesive tape. Leave the wound open but cover it with a sterile dressing. Free the foot and yucca board and wrap foot, yucca board, tubing and all with cotton batting and bandage liberally but not too tightly, being especially careful not to have any bandage pressure above the point of entrance of the catheter into the vein as this will obstruct the flow. Of equal importance is to prevent any traction or torsion of the tube which would be transmitted to the catheter. By fastening the tube firmly with adhesive tape where it emerges from the bandage any unexpected traction on the tube will be transmitted not to the catheter but to the bandages.

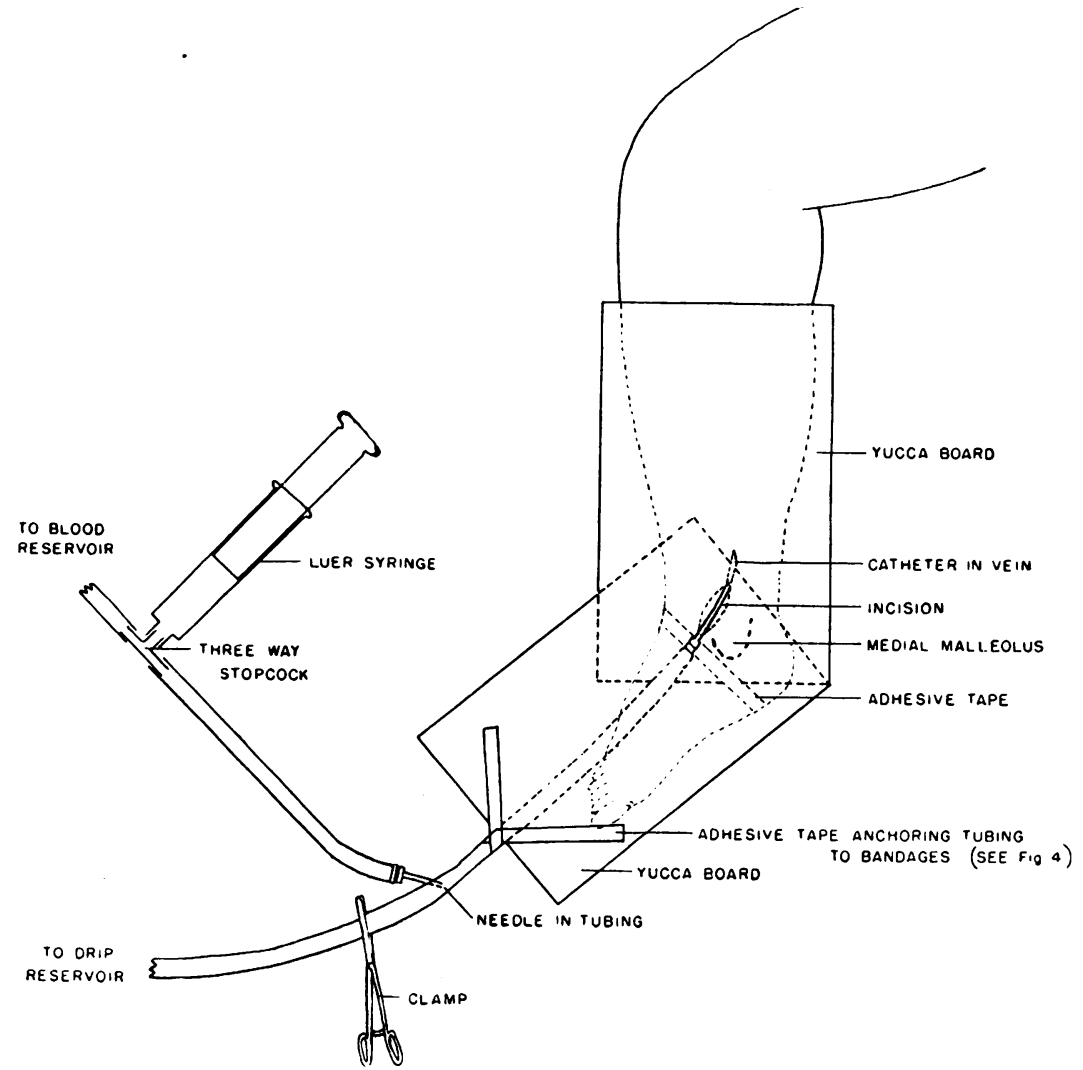

Fig. 2.-Diagramatic representation of continuous intravenous drip apparatus in use and the means by which a citrated blood transfusion may be given.

Adjustment of the rate of flow is accomplished by means of the screw clamp on the tubing above the Murphy drip bulb. When children or infants cry the venous pressure is temporarily raised. If the Murphy drip bulb is not kept three or four feet above the level of the ankle, during these rises of venous pressure a little blood may regurgitate into the catheter, coagulate and obstruct the system. If the drip bulb fills and the drops cannot be counted cleanse the tubing just below the drip bulb with iodine, and with a smaller needle and Luer syringe pierce the tube and slowly inject air into the bulb to displace the fluid. It should be injected just fast enough to stop the drops. It is well to keep the solution warm by means of an electric pad or hot water bottles. At low rates of flow this is of minor importance, 
Hypertonic solutions* are irritating to the wall of the vein and are prone to cause thrombosis. If they are used they should be alternated with jsotonic solutions. If the drip slows down unexpectedly disconnect the tube and aspirate the. catheter with a Luer syringe. This will often re-establish a free flow, apparently by dislodging a tiny flake of fibrin. If transfusions are to be given while the drip is in use the blood may be injected through the catheter. This is most conveniently accomplished by clamping the tubing an inch or two distal to the point where it emerges from the bandages. Sterilize the rubber tubing between the clamp and the

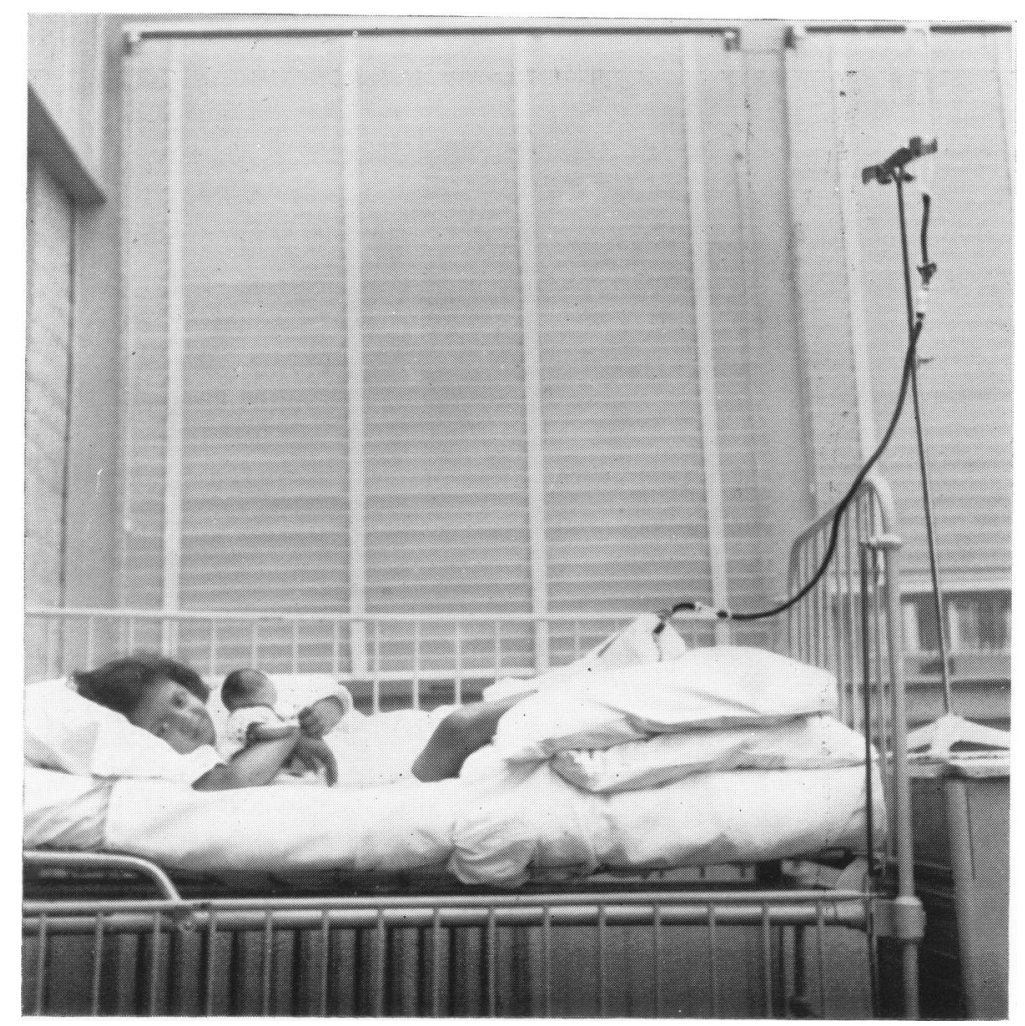

FIG. 3.-Continuous intravenous drip apparatus in operation. Note elevation of foot above level of base of heart.

bandages with tincture of iodine, puncture the sterilized tubing with the needle from the reservoir of citrated blood and permit the blood to run in either by gravity or by positive pressure with a Luer syringe connected with a three-way stopcock inserted between the needle and the tubing containing blood. The hole in the tubing will seal itself when the needle is removed. When the clamp is removed the drip will resume function (fig. 2).

* Five per cent. glucose, 0.9 per cent. sodium chloride, and 1/6 molar sodium lactate are all isotonic. When a combination of these is to be used only isotonic solutions should be mixed if the resultant solution is to remain isotonic, 


\section{Discussion}

Results with the continuous intravenous drip have been improved in recent months by the adoption of two simple points which diminish the tendency for blood to regurgitate into the catheter and coagulate. First the patient's foot is kept elevated by means of pillows several inches above the level of the base of the heart as much of the time as possible. Second, the nurse is instructed to open the stopcock to permit a maximum rate of flow for a few seconds every hour (fig. 3 and 4). When the drip is dis-

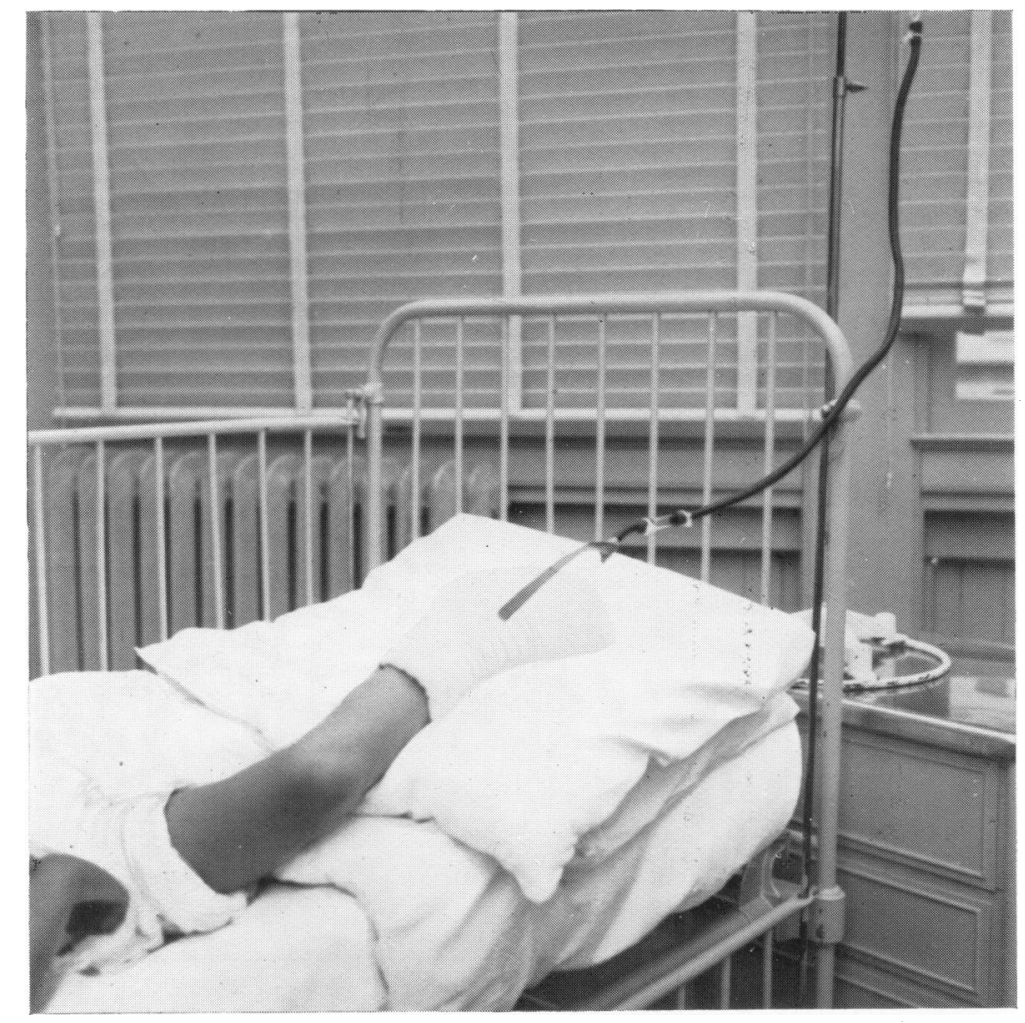

FIG. 4.-Continuous intravenous drip apparatus in operation. Note anchoring adhesive tape (stained darkly), liberal bandaging of foot and leg and absence of tight bandaging above the incision.

continued (and it can run for three days or longer if desired), the two ligatures are gently cut away from the vein and a fairly firm moist dressing applied in such a way that the edges of the wound are approximated. The vein probably 'becomes patent again eventually.

That the continuous intravenous drip has inherent dangers cannot be denied. They are: (1) sudden flooding of the circulation; (2) air embolism; (3) wound infection; (4) thrombosis and embolism. The first two are 
entirely preventable. The third, wound infection, may occur occasionally even when proper antiseptic precautions are taken. That infection is of infrequent occurrence is attested by the fact that we have not seen a single instance of it. Thrombosis and embolism probably occur more frequently than they are recognized. Rumold ${ }^{2}$ offers experimental evidence to support this view. Induration and tenderness over the vein have occasionally been noted proximal to the site of attachment of the apparatus which persists for several days after discontinuing the drip. This is more likely to occur if hyper- or hypotonic solutions are employed. Orr ${ }^{3}$ reported a case of pulmonary embolism following the use of an intravenous drip and gleaned several other instances from the literature. Hence a word of caution must be offered: if the flow should stop do not attempt to clear the obstruction by applying positive pressure. It is only permissible to aspirate gently and if this is unsuccessful either to remove the catheter and replace it with a new one or to choose another vein and make a fresh start.

After functioning well for several days the flow occasionally may gradually become less free and eventually stop. This is often caused by gradual swelling of the catheter, probably from absorption of water, as also occurs when the same type of catheter is left for several days in the pelvis of the kidney for continuous drainage and irrigations. Despite this disadvantage we prefer the catheter to a metal cannula because the former is much less apt to irritate the wall of the vein and lead to occlusion. Furthermore a considerable degree of freedom of motion of the leg and foot is permitted. This latter feature is of great importance from the standpoint of the comfort of the patient, in facilitating turning of the patient and other features of nursing care.

\section{REFERENGES}

1. Brush, J. M., Am. J. Dis. Child., Chicago, 1932, XLIV, 366.

2. Rumold, M. J., Arch. Surg., Chicago, 1935, XXX, 685.

3. Orr, T. G., Minnesota Med., St. Paul, 1935, XVIII, 778. 